\title{
A self-consistent model of $\Gamma-X$ mixing in GaAs/AlAs/GaAs quantum well structures using the quantum transmitting boundary method
}

\author{
J. P. Sun, R. K. Mains, K. Yang, and G. I. Haddad \\ Center for High-Frequency Microelectronics, Solid State Electronics Laboratory, Department of Electrical \\ Engineering and Computer Science, The University of Michigan, Ann Arbor, Michigan 48109
}

(Received 15 March 1993; accepted for publication 15 July 1993)

\begin{abstract}
We present a numerical study of the $\Gamma-X$ mixing in GaAs/AlAs/GaAs quantum well structures. A $\Gamma-X$ mixing model proposed by Liu [Appl. Phys. Lett. 51, 1019 (1987)] is extended to include the effects of self-consistency and nonzero transverse momentum. In the present model, the coupled Schrödinger equations for $\Gamma$ and $X$ electron envelope wave functions are solved self-consistently with Poisson's equation to calculate the electron transmission probability and wave functions, which lead to the current-voltage $(I-V)$ characteristics of single barrier and double barrier resonant tunneling diode structures. The quantum transmitting boundary method is employed in the model for numerical solution of the coupled Schrödinger equations, which proves to be very stable and efficient, even for large ( $>2000 \AA)$ structures. The features of $\Gamma-X$ mixing, such as the resonance/antiresonance in the transmission probability and the virtual bound states, are clearly demonstrated. Additional physical features are observed in the transmission probability and the wave functions under applied bias conditions. Our work shows that inclusion of transverse momentum, variable effective mass, and the self-consistent potential is important in the realistic modeling of $I-V$ characteristics for structures exhibiting $\Gamma-X$ coupling.
\end{abstract}

\section{INTRODUCTION}

The modeling of quantum well structures involving the $\mathrm{GaAs} / \mathrm{Al}_{x} \mathrm{Ga}_{1-x} \mathrm{As} / \mathrm{GaAs}(x>0.4)$ or $\mathrm{GaAs} / \mathrm{AlAs} / \mathrm{GaAs}$ systems is complicated by the fact that the electron transmission probability depends on both the $\Gamma$ and $X$ states and their interaction in the system. A simple envelope function approach, applied to $\Gamma$ and $X$ states separately, breaks down for the case of coupling between different valleys; thus researchers usually resort to more microscopic models. Several theoretical studies of $\Gamma-X$ mixing are based on the empirical tight-binding model, ${ }^{1-3}$ or pseudopotential calculations, ${ }^{4}$ or a combination with the envelope function approach. ${ }^{5}$ On the other hand, while these calculations have generated ample information about the energy band and revealed features of the mixing effects, they are not considered to be very suitable for routine modeling purposes, where flexibility and simplicity are desired for device analysis. Moreover, to our knowledge, no selfconsistent model of $\Gamma-X$ mixing has been carried out. On the other hand, $\mathrm{Liu}^{6}$ has recently introduced a coupling coefficient at the heterojunction interfaces to account for the $\Gamma-X$ scattering potential in an envelope function formulation to evaluate the flatband transmission probability. However, in order to assess the current-voltage $(I-V)$ and/or capacitance-voltage $(C-V)$ characteristics of device structures where $\Gamma-X$ mixing effects are significant, one must also include the effects of applied bias, temperature, doping profile, and space charge. For the space charge effects ${ }^{7}$ to be modeled, in particular, a self-consistent solution of the Schrödinger equation and Poisson's equation is desired..$^{8}$ In addition, knowledge of the electron wave functions in the structure is desirable because it gives not only device parameters of interest, but also renders more physical intuition of the problem. We have developed a numerical model for $\Gamma-X$ mixing in GaAs/AlAs/GaAs quantum well structures based on Liu's model but with the following extensions: (1) the coupled Schrödinger equations for the $\Gamma$ and $X$ state (envelope) wave functions are solved self-consistently with Poisson's equation to evaluate the effects of space charge and band bending on the current transport; (2) the carrier transverse momentum is incorporated in the formulation, which manifests the effects of conservation of transverse momentum during tunneling on the $I-V$ characteristics of the quantum well structures; ${ }^{9}$ (3) the quantum transmitting boundary method (QTBM) proposed by Lent and Kirkner ${ }^{10,11}$ is employed in the present model for the solution of the coupled Schrödinger equations and proved to be computationally very stable and efficient. Using the present model, the transmission probabilities and wave functions under flatband and biased conditions are calculated, which clearly demonstrate the main features of $\Gamma-X$ mixing and agree well with other existing models using tight-binding and psuedopotential calculations. Additional physical features pertaining to the resonant $\Gamma-X$ tunneling brought up by the self-consistent potential profile in the quantum well structures are observed. The calculated $I-V$ characteristics of the quantum well structures studied in this work are consistent with available experimental results and that from the other more microscopic models based on tight binding and pseudopotential calculations.

In Sec. II, the formulation of the model and the difference scheme used for numerical calculation are given. 


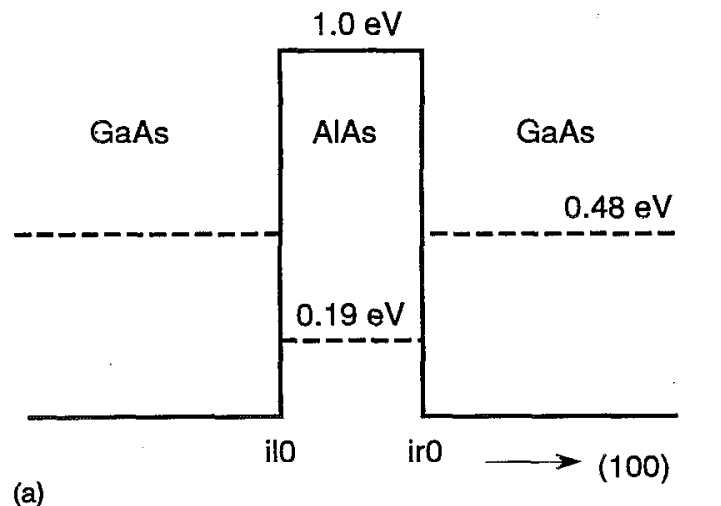

(a)

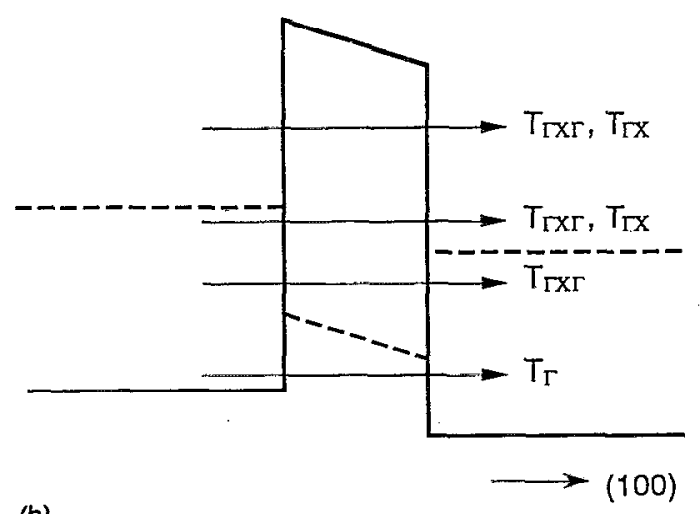

(b)

FIG. 1. (a) Band edge profiles for the GaAs/AlAs/GaAs structure in the growth direction. The $\Gamma$ and $X$ potentials are shown as solid and dashed lines, respectively. (b) The band profile under bias, showing the various transmission components.

The quantum transmitting boundary method as used in the present model is also described. The calculated results for the transmission probability under flatband and biased conditions and the $\Gamma$ and $X$ wave functions are presented in Sec. III. In Sec. IV, we discuss the significance of the self-consistent potential and transverse momentum in the calculation of the $I-V$ characteristics of a single barrier GaAs/AlAs/GaAs structure and a double barrier, resonant tunneling diode. Conclusions are given in Sec. V.

\section{MODEL AND NUMERICAL METHOD}

Consider Fig. 1(a), where the $\Gamma$ - and $X$-point potential profiles in the growth direction $x$ are shown schematically. The effective masses used in all the calculations are: $m_{\Gamma, \mathrm{GaAs}}^{*}=0.067 m_{0}, \quad m_{X, \mathrm{GaAs}}^{*}=1.30 m_{0}, \quad m_{\Gamma, \mathrm{AlAs}}^{*}=0.15 m_{0}$, and $m_{X, \mathrm{AlAs}}^{*}=1.10 m_{0}, m_{0}$ being the electron mass in free space. We will drop the asterisk denoting effective mass in the forthcoming formulas. The $\Gamma-X$ mixing at the heterointerfaces due to the lack of translational invariance is represented by a coupling constant $\alpha$ of $0.15 \mathrm{eV} \AA$ and a Delta function which turns on the coupling between the $\Gamma$ and $X$ waves at the heterointerfaces, labeled as $i l 0$ and $i r 0$. The intervalley scattering is modeled by the off-diagonal terms in a two by two potential matrix in the coupled Schrödinger equations:

$$
\begin{gathered}
{\left[\begin{array}{cc}
-\frac{\hbar^{2}}{2} \frac{\partial}{\partial x} \frac{1}{m_{\Gamma}} \frac{\partial}{\partial x} & 0 \\
0 & -\frac{\hbar^{2} \partial}{2 \partial x} \frac{1}{m_{x}} \frac{\partial}{\partial x}
\end{array}\right]\left[\begin{array}{l}
\psi_{\Gamma} \\
\psi_{X}
\end{array}\right]} \\
+\left[\begin{array}{cc}
V_{\Gamma}-E+\frac{\hbar^{2} k_{\|}^{2}}{2 m_{\Gamma}} & \alpha \delta(x) \\
\alpha \delta(x) & V_{X}-E+\frac{\hbar^{2} k_{\|}^{2}}{2 m_{x}}
\end{array}\right]\left[\begin{array}{l}
\psi_{\Gamma} \\
\psi_{X}
\end{array}\right]=0,
\end{gathered}
$$

where the effective masses are spatially varying according to the layered materials, $\mathbf{k}_{\|}$is the magnitude of the twodimensional transverse wave vector incorporated in the effective mass equations as formulated by Bastard, ${ }^{12}$ and $\delta(x)$ represents the Delta function defined previously. The matching conditions involving the wave functions and their derivatives at the heterojunction interfaces are given by

$$
\begin{gathered}
\psi_{\left.\Gamma\right|_{i 0^{-}} ^{i 0^{-}}}=\left.\psi_{\Gamma}\right|_{\substack{i 0^{+} \\
i 0^{+}}}, \\
\left.\psi_{X}\right|_{\substack{i 0^{-} \\
i 0^{-}}}=\left.\psi_{X}\right|_{\substack{i 0^{+} 0^{+} \\
i 0^{+}}},
\end{gathered}
$$

and

$\frac{\hbar^{2}}{2}\left[\left(\frac{1}{m_{\Gamma}} \frac{\partial \psi_{\Gamma}}{\partial x}\right)_{\substack{i 0^{-} \\ i 0^{-}}}-\left(\frac{1}{m_{\Gamma}} \frac{\partial \psi_{\Gamma}}{\partial x}\right)_{\substack{i 0^{+} \\ i 0^{+}}}\right]+\left.\alpha \psi_{X}\right|_{\substack{i \neq 0 \\ i r 0}}=0$,

$$
\frac{\hbar^{2}}{2}\left[\left(\frac{1}{m_{X}} \frac{\partial \psi_{X}}{\partial x}\right)_{\substack{i 0^{-} \\ i 0^{-}}}-\left(\frac{1}{m_{X}} \frac{\partial \psi_{X}}{\partial x}\right)_{\substack{i 0^{+} \\ i 0^{+}}}\right]+\left.\alpha \psi_{\Gamma}\right|_{\substack{i \neq 0 \\ i \sim 0}}=0
$$

The discretization of Eq. (1) should preserve the property of charge and current conservation in the system. It is straightforward to verify that the following difference formulas we propose for the wave functions ensure current continuity for any spatial index $i$ in the structure:

$$
\begin{aligned}
\psi_{\Gamma, i+1}= & \left(\frac{2 m_{\Gamma, i} \Delta x^{2}\left[V_{\Gamma, i}+\frac{\hbar^{2} k_{\|}^{2}}{2}\left(1 / m_{\Gamma, i}-1 / m_{\Gamma, c}\right)-E\right]}{\hbar^{2}}\right. \\
& \left.+1+\frac{m_{\Gamma, i}}{m_{\Gamma, i-1}}\right) \psi_{\Gamma, i}-\frac{m_{\Gamma, i}}{m_{\Gamma, i-1}} \psi_{\Gamma, i-1} \\
& +\frac{2 \Delta x^{2} m_{\Gamma, i}}{\hbar^{2}} \alpha \delta_{i} \psi_{X, i,}
\end{aligned}
$$




$$
\begin{aligned}
\psi_{X, i+1}= & \left(\frac{2 m_{X, i} \Delta x^{2}\left[V_{X, i}+\frac{\hbar^{2} k_{\|}^{2}}{2}\left(1 / m_{X, i}^{*}-1 / m_{X, c}^{*}\right)-E\right]}{\hbar^{2}}\right. \\
& \left.+1+\frac{m_{X, i}}{m_{X, i-1}}\right) \psi_{X, i}-\frac{m_{X, i}}{m_{X, i-1}} \psi_{X, i-1} \\
& +\frac{2 \Delta x^{2 m_{X, i}}}{\hbar^{2}} \alpha \delta_{i} \psi_{\Gamma, i}
\end{aligned}
$$

where the subscript $c$ of the effective masses denotes the masses in the contact regions. One-dimensional, steadystate solutions of the Schrödinger equation are usually obtainable by assuming a wave function of known form at a - boundary and integrating through the structure according to a recursion formula toward the other boundary, followed by an appropriate normalization. However, when attempting the $\Gamma-X$ mixing problems, this approach fails due to the exponential behavior of the electron states in the barrier. For example, keeping an exponentially growing wave during computation is likely to result in the loss of significant digits for the other decaying or traveling wave.

The QTBM we adopted in this work, on the other hand, enforces all the boundary conditions as well as the interface matching conditions during the solution process and the wave functions at every discretized point in the structure are solved simultaneously, thereby ruling out nonphysical results. Structures greater than $2000 \AA$ A, described in Sec. IV, have been simulated by sovling Poisson's equation and the coupled Schrödinger equations selfconsistently, using the QTBM for the solution of the coupled Schrödinger equations without encountering numcrical instability. For the present mixing problem, four boundary conditions were derived from the assumption that there are only $\Gamma$ electrons incident from the left hand side (lhs) boundary, and that no reflections occur at the right hand side (rhs) boundary:

$$
\begin{aligned}
& \psi_{\Gamma, 1} \exp \left(-j k_{\Gamma} \Delta x\right)-\psi_{\Gamma, 2}=-2 j \sin \left(k_{\Gamma} \Delta x\right), \\
& \psi_{X, 1} \exp \left(-j k_{X} \Delta x\right)-\psi_{X, 2}=0, \\
& \psi_{\Gamma, N} \exp \left(-j k_{\Gamma}^{\prime} \Delta x\right)-\psi_{\Gamma, N-1}=0, \\
& \psi_{X, N} \exp \left(-j k_{X}^{\prime} \Delta x\right)-\psi_{X, N-1}=0,
\end{aligned}
$$

where $j=\sqrt{-1}, \Delta x$ is the stepsize, and $N$ is the point index of the structure.

Note that in the present problem the $k$ 's are in general complex wave numbers for electrons with a spectrum of incident energy scattered by the spatially varying $\Gamma$ and $X$ point potentials. A banded matrix equation of order $2 N$ with a bandwidth 5 of the complex coefficient matrix is then constructed by combining Eqs. (6)-(11) and solved numerically for the $\Gamma$ and $X$ wave functions using a banded matrix solution procedure.

The calculation for the self-consistent potential and charge distributions in this work is performed by solving Poisson's equation for the $\Gamma$ point potential only. The $X$ point potential is assumed to have the same band bending as in the $\Gamma$ point potential. That is, we assume that the separation between the $\Gamma$ and $X$ potentials is unchanged by the space charge. The solution for self-consistency involves a Thomas-Fermi calculation and two quantum calculations. In the Thomas-Fermi approximation, carrier concentrations are obtained assuming constant Fermi levels within the device contact regions, determined using equilibrium Fermi-Dirac statistics. An extension of the JoyceDixon approximation is used to evaluate the Fermi integral at low temperatures. In the barrier/quantum well region(s), the $X$ valley bound state charge is determined by an eigenvalue solution of the time-independent Schrödinger equation for the $X$ point potential in the single barrier case, whereas in the case of an RTD structure, the $\Gamma$ and $X$ bound states are calculated separately using the $\Gamma$ and $X$ potentials independently, and the space charge in the AlAs $X$ valleys is neglected for simplicity. The space charge in the GaAs $\Gamma$ valley is then coupled to the Thomas-Fermi calculation to achieve a self-consistent potential for a particular bias voltage across the device structure. The current density is calculated from the $\Gamma$ and $X$ wave functions obtained from the coupled Schrödinger equations for a self-consistent potential under a specific bias and a given transverse momentum. In the calculated result with nonzero transverse momentum, $k_{\|}$is taken to be $2.0 \times 10^{6} \mathrm{~cm}^{-1}$, which is approximately $20 \%$ of the magnitude of the Fermi wave vector for the doping level and temperature in the structures being modeled. Details of the self-consistent method and the associated numerical schemes outlined above have been described elsewhere. ${ }^{13,14}$ In the numerical computation, all input parameters were assumed to be accurate to four significant digits in order to show the sharp resolution of the transmission probability.

\section{III. $\Gamma-X$ MIXING FEATURES}

We demonstrate in this section the main features of the $\Gamma-X$ mixing in a single barrier GaAs/AlAs/GaAs structure, using the present model without invoking selfconsistency, as illustration and for convenience in comparing with the results from other existing models. The barrier thickness used in this section is $50 \AA$. The tunneling mechanisms can be identified for the structure under consideration as shown in Fig. 1(b), where $\Gamma_{\Gamma X \Gamma}$ stands for the transmission probability through the $\Gamma-X-\Gamma$ channel, and $T_{\Gamma X}$ for the transmission via the $\Gamma-X$ channel. Phenomenologically, for $T_{\Gamma X \Gamma}$, an incident electron from the $\Gamma$ valley of the lhs GaAs emitter is scattered into the $X$ valley in AlAs, transferring in both real and momentum space at the interface ( $i l 0$ ); a similar $X-\Gamma$ transfer takes place at $i r 0$. The relative amplitude of $\psi_{X}$ and $\psi_{\Gamma}$ in AlAs is an indication of the probability of the electron being scattered into the $X$ valley. When the incident energy is degenerate with a bound state energy associated with the $X$ valley in AlAs, strong resonances with near unity transmission occur due to constructive interference between the $\Gamma$ and $X$ waves and these bound states behave like virtual states, allowing the electrons to pass through. Antiresonances are also observed because of destructive interference. The transmis- 

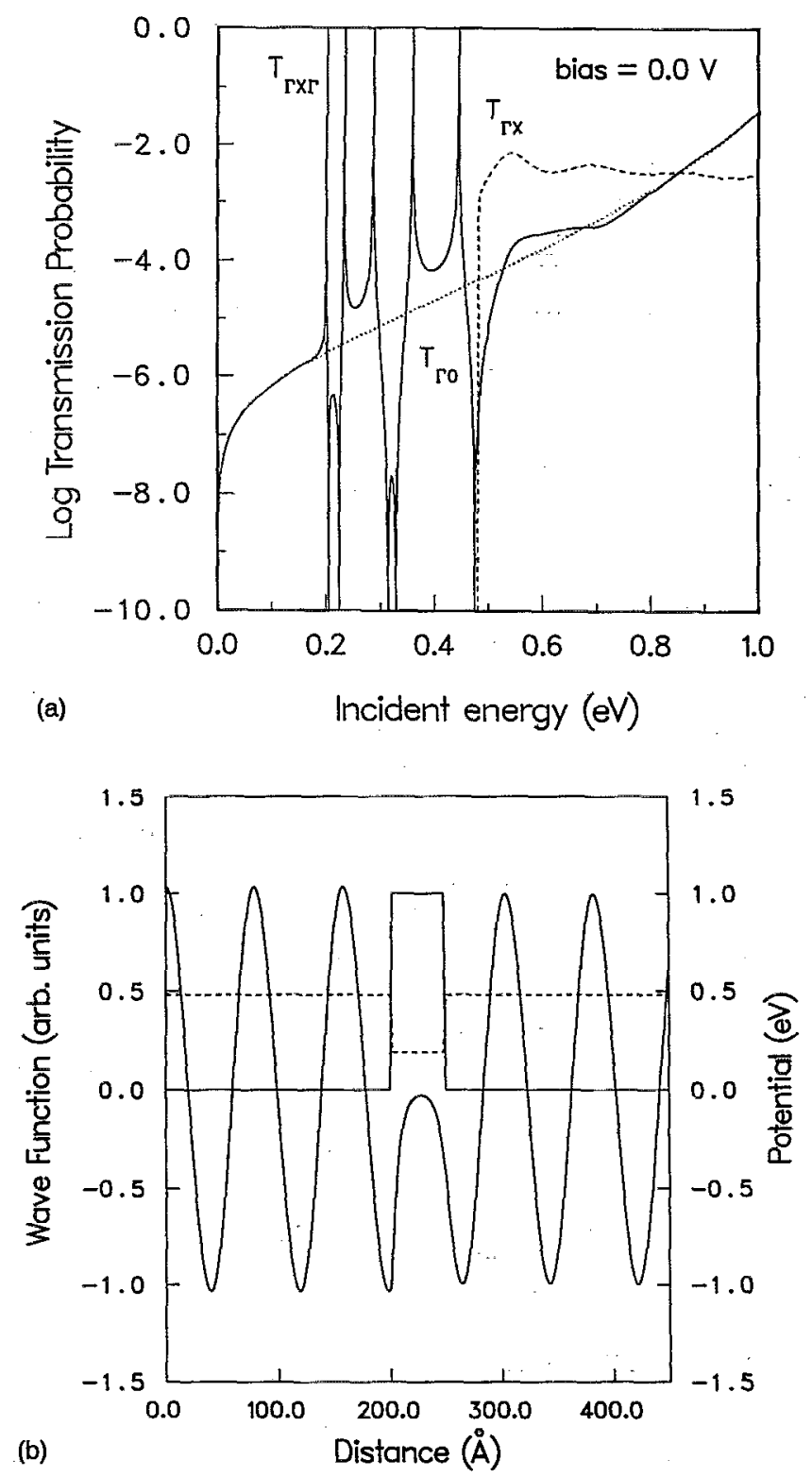

sion probability is defined to be the ratio of the outgoing electron flux at the rhs to the incoming flux at the lhs,

$$
\begin{aligned}
& T_{\Gamma X \Gamma}=\left|C_{\Gamma}^{\text {ehs }} / A_{\Gamma}^{\text {lhs }}\right|^{2}\left(k_{\Gamma}^{\text {rhs }} m_{\Gamma}^{\text {lhs }}\right) /\left(k_{\Gamma}^{\text {lhs }} m_{\Gamma}^{\text {rhs }}\right), \\
& T_{\Gamma X}=\left|C_{X}^{\text {chs }} / A_{\Gamma}^{\text {lhs }}\right|^{2}\left(k_{X}^{\text {rhs }} m_{\Gamma}^{\text {lhs }}\right) /\left(k_{\Gamma}^{\text {lhs }} m_{X}^{\text {rhs }}\right),
\end{aligned}
$$

where the $C$ 's and $A$ 's are the incident and transmitted wave amplitudes, respectively. In Fig. 2(a), five distinct resonant peaks and antiresonant dips appear on the calculated $T_{\Gamma X \Gamma}$ transmission curve in the flatband case at energies close to the calculated bound state energies of the $X$ point potential, signifying strong coupling. The coupling effect is otherwise weak. The $T_{\Gamma X}$ turns up at the energy of the top of the $X$ valley in GaAs. It is relatively small since the propagating $X$ mode is generated only by the small coupling at the interfaces from the incident $\Gamma$ wave. Also shown in the figure for comparison is $T_{\Gamma 0}$, the transmission for the $\Gamma$-only potential when the coupling is turned off, having the well-known exponential dependence on the in-

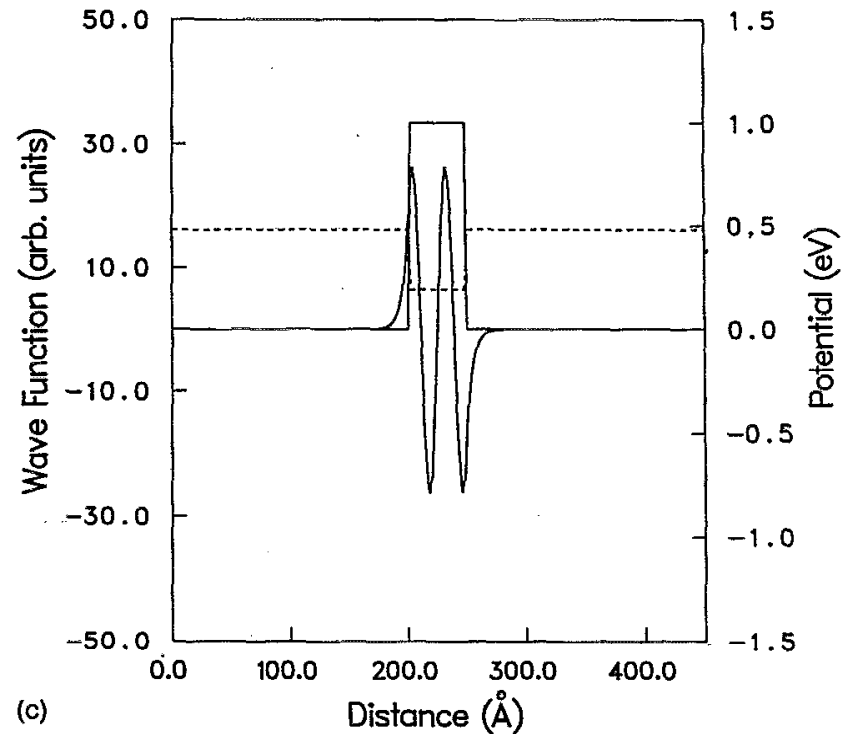

FIG. 2. (a) Transmission probability vs electron incident energy under flat-band condition. Solid line: $T_{\Gamma X \Gamma}$, dashed line: $T_{\Gamma X}$, and dotted line: $T_{\Gamma 0}$ with the zero coupling. (b) The $\Gamma$ wave function at the fourth transmission peak with an electron incident energy of $359.1 \mathrm{meV}$. (c) The $X$ wave function at the same transmission peak.

cident energy. We have taken the emitter $\Gamma$ point energy as zero energy reference in all the calculations.

The $\Gamma$ and $X$ wave functions at the transmission peaks all have similar behavior, with the $\Gamma$ waves propagating and the $X$ waves exhibiting the quasi-bound state character. The real parts of the $\Gamma$ and $X$ wave functions at the fourth transmission peak, with an electron incident energy of $359.1 \mathrm{meV}$, are plotted in Fig. 2(b) and 2(c), respectively, as an example. The traveling mode of the $\Gamma$ wave is obvious on both sides of the barrier, with equal amplitude. The behavior and magnitude of the $\Gamma$ wave at the interfaces reflect the interface conditions set up in Eqs. (2)(5), while its amplitude squared in the barrier may be considered as the probability of an electron remaining in the $\Gamma$ valley after being scattered. The $X$ wave, as expected, does not vanish at the $X$ valley boundaries as the true bound states, but rather it shows discernible penetration into the walls of the $X$ valley in GaAs. This is a conse- 

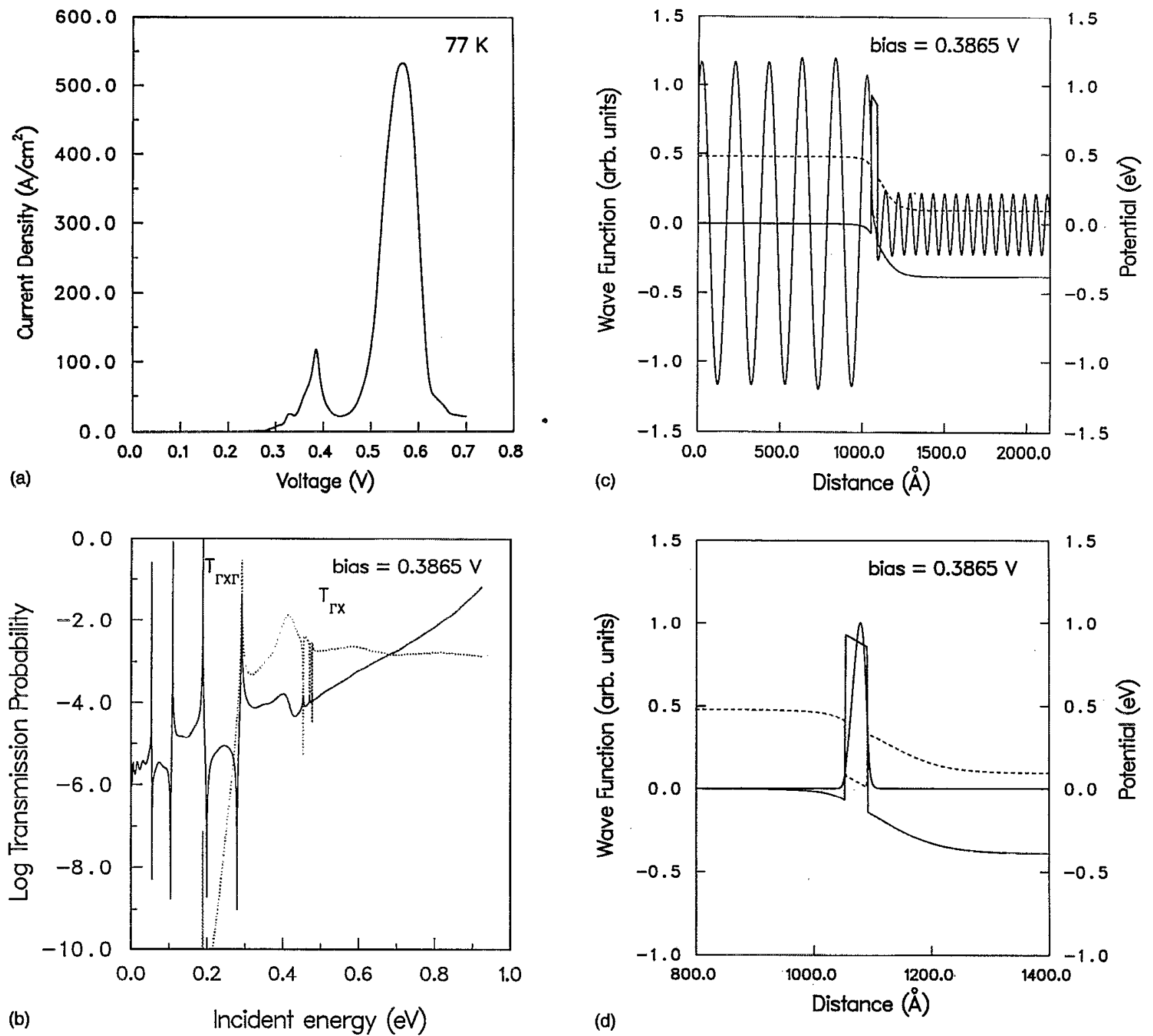

FIG. 3. (a) Calculated $I-V$ characteristics of the single barrier structure at $77 \mathrm{~K}$. (b) Transmission probability vs electron incident energy at the peak voltage of $0.3865 \mathrm{~V}$. Solid line: $T_{\mathrm{r} X \Gamma}$, dotted line: $T_{\Gamma X}$. (c) The $\Gamma$ wave function (solid line) at the first transmission peak with an electron incident energy of $53.35 \mathrm{meV}$. (d) The $X$ wave function (solid line) at the same peak. Only the central part of the device is shown. In both (c) and (d), the $\Gamma$ potential is also shown in solid line, and the $X$ potential in dotted line.

quence of the interaction between the $\Gamma$ and $X$ states. Away from these peaks, however, the wave functions (not shown) only exhibit weak coupling, consistent with the transmission curve.

We have also performed the transmission calculation using the transfer matrix method for the flatband case as done in Ref. 6 and an excellent agreement was found in comparison with the numerical solution using the present model.

The transmission probability and the wave forms under biased conditions will be shown and discussed when we present self-consistent calculations for practical quantum well structures described next.

\section{RESULTS WITH SELF-CONSISTENCY}

We now examine a single barrier structure with a $40 \AA$ AlAs barrier, sandwiched by $50 \AA$ nominally undoped GaAs spacers, where a background N-type doping of $10^{14} \mathrm{~cm}^{-3}$ was assigned. The GaAs contact regions (emitter and collector) are $\mathrm{N}^{+}$-type doped to $10^{18} \mathrm{~cm}^{-3}$ with a thickness of $1000 \AA$ each, sufficient to allow the potential to reach equilibrium in the contact regions. This structure is very similar to that in the experimental observation of negative differential resistance (NDR) reported by Beresford et $a l^{15}$

In evaluating the $I-V$ characteristics of the structure, 


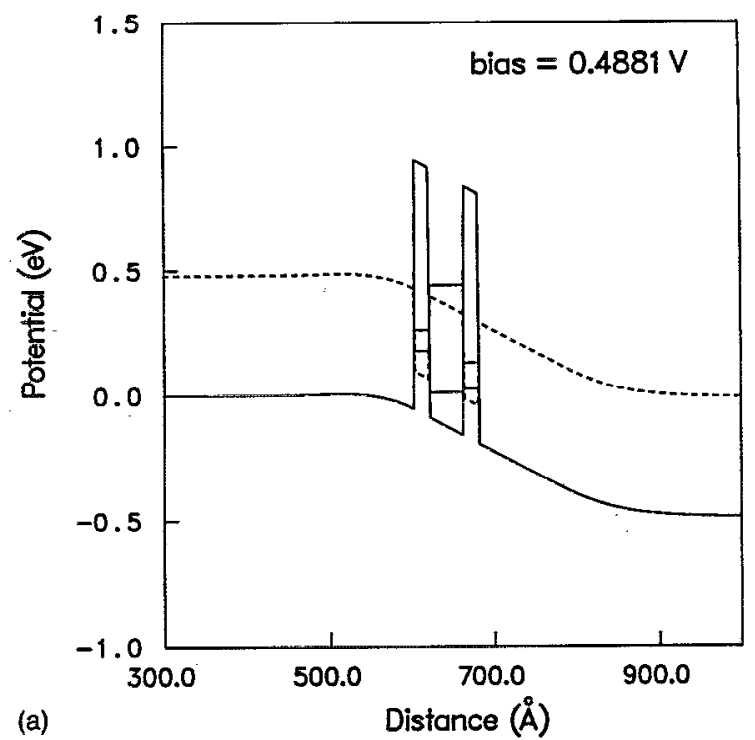

(a)

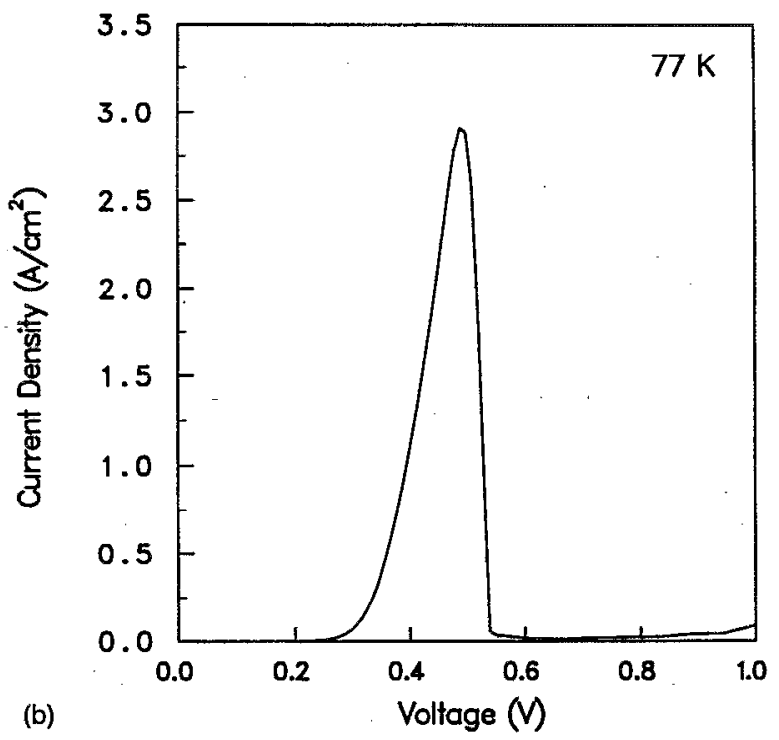

we have noted the following effects: (1) The incident $\Gamma$ electrons from the emitter see an effeclively lowered $\Gamma$ barrier, and a triangular $X$ point barrier is formed and it affects both the $\Gamma$ and $X$ electron transport. Additional reflection and interference occur at the multiple barriers and interfaces. (2) Much of the applied voltage is taken up by the accumulation and depletion regions, which reduces the rate of downward movement of the $X$ valley quasi-bound levels with increasing bias voltage. (3) Space charge in the $X$ valley states in the case of the single barrier structure modifies the potential profile which in turn affects the electron wave functions. (4) The effective mass difference in the emitter and barrier has a significant effect on the device $I$-V characteristics, as discussed by Ohno et al. ${ }^{9}$ For these effects to be built into a model, self-consistency, the electron transverse momentum, and variable effective mass must be incorporated in a formulation, such as implemented in the present model.

We have also considered the effects of the formation of a quantized accumulation layer. When it is formed at the

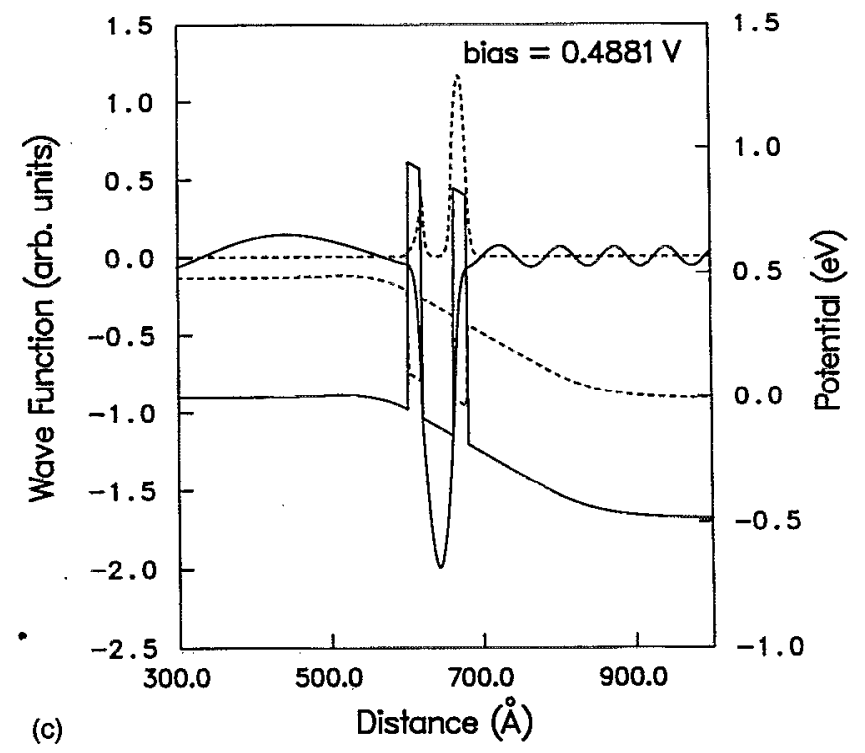

FIG. 4. (a) The band profiles of the RTD structure at the peak voltage of $0.4881 \mathrm{~V}$. Solid line: $\Gamma$ potential. Dashed line: $X$ potential. The bound statcs in the quantum wells are also shown in solid lines. (b) Calculated $I-V$ characteristic of the double barrier (RTD) structure at $77 \mathrm{~K}$. (c) Calculated $\Gamma$ and $X$ wave functions for electron incident at the energy of first transmission peak $(12.65 \mathrm{meV})$. The $\Gamma$ potential and wave function are shown in solid lines, the $X$ potential and wave functions are shown in dashed lines. Only the central part of the device is shown.

emitter side near the barrier with the emitter negatively biased, the electrons residing in the quantized levels in the accumulation region may tunnel through the $X$ valley states in the AlAs barrier and contribute to the current. This effect is estimated in the present model and it was found that, for the structures under consideration, the charge in the quantized levels there is not significant until fairly high bias since the structure has heavily doped contact layers. This effect is therefore neglected in the further discussions.

In Fig. 3(a) we show the modeled $I-V$ characteristics of the single barrier structure. It is calculated with $\alpha=0.175 \mathrm{eV} \AA$, the $X$ valley height in AlAs, $V_{b x, \text { AlAs }}=0.15$ $\mathrm{eV}$, and $k_{\|}=2.0 \times 10^{6} \mathrm{~cm}^{-1}$ at $77 \mathrm{~K}$. The peak current density is $117.4 \mathrm{~A} \mathrm{~cm}^{-2}$ at the peak voltage of $0.3865 \mathrm{~V}$. The peak-to-valley ratio is approximately 5 . In comparison with the experimental observation of negative differential resistance $(N D R)^{15}$ in a single barrier structure, the present calculation, although not an accurate rep- 
lica of the experimental $I-V$ curve, has modeled the main features of the characteristics.

Figure 3(b) shows the calculated transmission probabilities $T_{\Gamma X \Gamma}$ and $T_{\Gamma X}$ as functions of electron energy under the peak voltage of $0.3865 \mathrm{~V}$. The transmission coefficient has four major peaks at 53.35, 109.2, 188.4, and 291.1 $\mathrm{meV}$. Comparing with the bound state energies at 53.21, $108.8,186.3$, and $285.7 \mathrm{meV}$, calculated from the $X$-point potential at this bias voltage, the transmission peaks are shifted approximately by 0.1 up to $5 \mathrm{meV}$ upward in cnergy due to the coupling of the $\Gamma$ and $X$ states. Note that the first transmission peak is smaller compared with the second and third peaks as the $X$ electron at that energy sees an additional triangular $X$ barrier, which reduces the transmitted $\Gamma$ wave. This can be seen from the $\Gamma$ and $X$ wave functions at the first $X$ bound state energy $(53.35 \mathrm{meV})$ shown respectively in Figs. 3(c) and 3(d), in which the potential profiles are also displayed. The fourth transmission peak in Fig. 3(b) is considerably smaller than the other peaks probably because the associated $X$ state is much less localized. The transmission through the first $X$ valley bound state in the barrier makes the most important contribution to the peak current since the other $X$ bound states lie higher in energy and the Fermi distribution function at the emitter has much less weight at these energies in supplying incident electrons. Another feature associated with the peak current is that it occurs when the first $X$ bound state level in the AlAs lies below the Fermi level at the emitter, being $53.7 \mathrm{meV}$ in our calculation for an emitter doping of $10^{18} \mathrm{~cm}^{-3}$ at $77 \mathrm{~K}$. The separation between these energy levels, however, is smaller than the energy separation obtained in Ref. 9. This discrepancy can bc attributed to the nonzero transverse momentum added, which virtually lowered the potential by approximately 15 $\mathrm{meV}$ in the Hamiltonian of the Schrödinger equations, and to the upward extension of the distribution function at the nonzero temperature. The next current peak of the $I-V$ curve is much higher and occurs at approximately $0.2 \mathrm{~V}$ away from the first peak voltage since, in our selfconsistent calculation, the accumulation and depletion laycrs take up approximately $75 \%-80 \%$ of the applied voltage and the second resonant $X$ valley state does not move down quickly enough to give significant contribution to the current at lower bias.

The method was also applied to model a double barrier RTD structure. The structure has two $20 \AA$ AlAs barriers, a $40 \AA$ GaAs quantum well, and $100 \AA$ emitter and collector spacers $\mathrm{N}$ doped to $10^{14} \mathrm{~cm}^{-3}$. The emitter and collector contacts are both $500 \AA$ and $\mathrm{N}^{+}$doped to $10^{18}$ $\mathrm{cm}^{-3}$. The energy band profiles at the peak voltage $(0.4881$ $V)$ of its $I-V$ characteristic are shown in Fig. 4(a). Also shown are the positions of the $\Gamma$ (in GaAs) and $X$ (in AlAs) bound state levels. This structure is similar to that used in Ref. 2 for the tight-binding calculation. The calculated $I-V$ curve using the present model is plotted in Fig. 4 (b). The peak current density is $2.908 \times 10^{5} \mathrm{~A} \mathrm{~cm}^{-2}$ and the valley current density is $3.226 \times 10^{3} \mathrm{~A} \mathrm{~cm}^{-2}$, yielding a peak-to-valley current ratio of approximately 90 . Note that the current peaks when the first $\Gamma$ bound state in the GaAs well $(12.65 \mathrm{meV}$ ) lies considerably below the Fermi level in the emitter, and that the $X$ bound states in the AlAs barriers are located higher relative to the first $\Gamma$ bound state at the peak voltage. As a result, the current peak of the RTD structure is only slightly related to the $X$ valley states, in contrast to the case of the single barrier structure. Moreover, the GaAs quantum well has a larger voltage drop than the AlAs barrier, lowering the first $\Gamma$ bound state at a faster rate than for the $X$ bound states. In Fig. 4(c) the calculated $\Gamma$ and $X$ wave functions for the electron incident at the energy of the first transmission peak $(12.65 \mathrm{meV})$ are shown. The resonance is clearly illustrated. Although the $X$ states may not be the main cause for the peak current, they do contribute to the valley current, giving rise to a more realistic peak-to-valley ratio. The valley current in an RTD is also associated with other important scattering processes, such as phonon-assisted tunneling, which have not been modeled in this work; however, the present model has given a more realistic estimate of the $I-V$ characteristics. We note that a very accurate prediction of the $I-V$ characteristics of resonant tunneling devices is still a formidable task with the present status of quantum device modeling based on the single electron theory as many other scattering mechanisms have not been treated properly within a unified approach. Even with a quantum kinetic model based on the Wigner distribution function formalism, difficulties remain to be resolved. ${ }^{16}$

\section{CONCLUSIONS}

We have presented a self-consistent numerical model for the $\Gamma-X$ mixing effects in GaAs/AlAs/GaAs quantum well structures. The model has the virtues of being conceptually simple, computationally very stable and efficient, and flexible in terms of its application to device modeling. Major $\Gamma-X$ mixing effects derived from the calculated wave functions are clearly demonstrated. Additional features of the transmission properties due to the inclusion of selfconsistency and transverse momentum are observed and discussed, which has lead to more realistic $I-V$ characteristics of single barrier and double barrier quantum well structures. This model may have extended applications for various heterojunction and quantum well structures where the $\Gamma-X$ coupling effects are significant.

\section{ACKNOWLEDGMENTS}

The authors would like to thank Professor J. Singh, Dr. J. R. East, and Dr. V. Sankaran for helpful discussions. This work was supported by the Army Research Office under the URI program Grant No. DAAL03-92-G-0109.

\footnotetext{
${ }^{\prime}$ C. Maihiot, T. C. McGill, and J. N. Schulman, J. Vac. Sci. Technol. B 1, 439 (1983) and the references therein.

${ }^{2}$ T. B. Boykin, J. P. A. van der Wagt, and J. S. Harris, Jr., Phys. Rev. B 43, 4777 (1991).

${ }^{3}$ T. B. Boykin and J. S. Harris, Jr., J. Appl. Phys. 72, 988 (1992).

${ }^{4}$ A. C. Marsh, Semicond. Sci. Technol. 1, 32 (1986).

${ }^{5}$ T. Ando and H. Akera, Phys. Rev. B 40, 619 (1989), and the references therein.

${ }^{6}$ H. C. Liu, Appl. Phys. Lett. 51, 1019 (1987); Superlattices and Microstructures 7, 35 (1990).
} 
${ }^{7}$ V. J. Goldman, D. C. Tsui, and J. E. Cunningham, Phys. Rev. B 35, 9387 (1987).

${ }^{8}$ H. Ohnishi, T. Inata, S. Muto, N. Yokoyama, and A. Shibatomi, Appl. Phys. Lett. 49, 1248 (1986).

${ }^{9}$ H. Ohno, E. E. Mendez, and W. I. Wang, Appl. Phys. Lett. 56, 1793 (1990)

${ }^{10}$ C. S. Lent and D. J. Kirkner, J. Appl. Phys. 67, 6353 (1990).

${ }^{11}$ W. R. Frensley, Superlattices and Microstructures 11, 347 (1992).

${ }^{12}$ G. Bastard, Wave Mcchanics Applied to Semiconductor Heterostruc- tures, Les Editions de Physique (Les Ulis, France, 1988), p. 74.

${ }^{13}$ R. K. Mains, J. P. Sun, and G. I. Haddad, Appl. Phys. Lett. 55, 371 (1989).

${ }^{14}$ J. P. Sun, R. K. Mains, W. L. Chen, J. R. East, and G. I. Haddad, J. Appl. Phys. 72, 2340 (1992).

${ }^{15}$ R. Beresford, L. F. Luo, W. I. Wang, and E. E. Mendez, Appl. Phys. Lett. 55, 1555 (1989).

${ }^{16}$ R. K. Mains and G. I. Haddad (unpublished). 